\title{
Cross Skin Reactivity to Tyrosine Kinase Inhibitors in a Patient with Chronic Myelogenous Leukemia
}

\author{
V. Broshtilova ${ }^{*}$, M. Balabanova \\ Department of Dermatology and Venereology, Faculty of Medicine, Sofia Medical University, Sofia, Bulgaria. \\ Email: "broshtolova@mail.bg
}

Received June $9^{\text {th }}, 2013$; revised July $10^{\text {th }}, 2013$; accepted July $18^{\text {th }}, 2013$

Copyright (c) 2013 V. Broshtilova, M. Balabanova. This is an open access article distributed under the Creative Commons Attribution License, which permits unrestricted use, distribution, and reproduction in any medium, provided the original work is properly cited.

\begin{abstract}
Tyrosine kinase inhibitors (TKI) targeting the bcr-abl protein, c-kit and the platelet-derived growth factor receptors, are significant part of the pathogenic therapy of chronic myelogenous leukemia. A broad spectrum of cutaneous side effects has been described with the clinical use of imatinib mesylate, ranging from various acute rashes to toxic epidermal necrolysis. Herein, a case of cross skin toxicity to TKI in a patient with chronic myelogenous leukemia is presented. In the course of imatinib mesylate therapy the patient developed a grade 4 diffuse lichenoid drug eruption. Six months after switching to nilotinib, hyperpigmented macules and patches spread over his trunk and extremities. To date, few cases of cross skin reactivity to imatinib and nilotinib have been described, none of which showing different clinical phenotypes. Further understanding of the underlying mechanisms and leading to the development of skin rashes from different class of TKI is important to highlight new drug targets and modify the current therapies to a level of maximal efficacy.
\end{abstract}

Keywords: Cross Toxicity; Lichenoid Drug Reaction; Hyperpigmentation

\section{Introduction}

Imatinib mesylate has revolutionized the treatment of chronic myeloid leukemia (CML) and has been recently approved as first line therapy in CML patients. Many patients receiving imatinib mesylate experience hematological and non-hematological side effects. Clinical intolerance occurs when the toxicity of a medication outweighs its clinical benefit. Frequent non-hematological adverse effects are nausea, musculoskeletal pain, superficial edema, skin rashes and muscular cramps. The prevalence and their relationship with the dose suggest that they are the direct pharmacological effect of imatinib [1]. Early recognition of clinical intolerance to BCR-ABL inhibitors used for CML is important for maximizing patient benefit.

Among skin changes caused by imatinib, the most common adverse effects are superficial skin edema, rashes, hypopigmentation and pruritus [2]. Rarely does it lead to severe epidermal necrolysis, acute generalized exanthematous pustulosis, hyperpigmentation and lichenoid eruption $[3,4]$. The molecular mechanism for the skin changes is still obscure. Patients who experience unresponsive grade 3 or any grade 4 nonhematologic side effects may

${ }^{*}$ Corresponding author. require discontinuation and switch to second-line therapies, such as dasatinib or nilotinib, after identification of intolerance.

Nilotinib (formerly AMN 107), a highly selective bcrabl TKI, is effective in patient with Philadelphia chromosome positive CML, following resistance or intolerance to imatinib [5]. Nilotinib is considered potent and safe with minimal cross-reactivity to imatinib. Neutropenia and thrombocytopenia may be observed in $29 \%$ of patients, together with mild pleural or pericardial effusions. Main precaution of the drug usage is the QT prolongation warning [6]. Only mild and easily-managed cutaneous side effects have been implicated to this drug [7, 8].

Herein, a case of cross skin intolerance to tyrosine kinase inhibitors in a patient with CML is presented.

\section{Case Report}

A 61-year-old man presented with weakness and fatigue in November 2007. He had hepatomegaly of $4 \mathrm{~cm}$ and splenomegaly of $2 \mathrm{~cm}$. Bone marrow aspiration was suggestive of chronic myeloproliferative disorder. Leukocyte alkaline phosphatase score was zero. His cytogenetic study was positive for Philadelphia chromosome. He 
began imatinib mesylate $600 \mathrm{mg}$ daily in May 2008 and achieved complete hematological response within 1 month. Eight weeks after the first administration of the drug a wide spread eruption of lichenoid papules and plaques occurred [Figure 1]. The skin changes were very pruritic and abruptly disseminated throughout the trunk, upper and lower extremities to constellate generalized exfoliative dermatitis. He complained of profound malaise, weight loss, fever up to $39^{\circ} \mathrm{C}$, and nausea. All microbiologic cultures were repeatedly negative. The diagnosis of drug-induced lichenoid eruption was concluded, and the offended drug was discontinued. A short-term systemic corticosteroid therapy of $40 \mathrm{mg}$ 6-methylprednisolon was introduced to cope with the skin changes, which resolved completely in 3 weeks. Therapeutic challenge evolved with patient's pathogenetic CML therapy. He received hydroxyurea and interferon $5 \mathrm{MIU} /$ day, but there was no hematological remission and cytogenetical response. Therefore, in January 2010 nilotinib 400 mg/day was started. He achieved complete hematological response within two months. The cytogenetic response was partial at third months and complete at fifth months. In May 2010 a symmetrical pruritic hyperpigmented papular eruption with areas of confluence developed on his extremities, abdomen, trunk, back, and neck. The skin changes were most prominent on the extremities and lateral aspects of trunk where they formed confluent reticulate dark-brown hyperpigmented plaques [Figure 2] in a midline confluent manner. Skin biopsy showed parakeratosis, acanthosis, interface dermatitis with few apoptotic keratinocytes in the lower epidermal layers, and pigment incontinence with abundance of melanophages in the papillary dermis. A mild perivascular lympho-histiocytic infiltrate was seen in the upper dermis. Immunohistochemistry staining ruled out cutaneous histiocytoses [Figure 3]. The patient did not have any significant sun exposure and did not use systemic steroids during the course of his disease. He refused discontinuation of the drug, since the itchy skin lesions were tolerated well with topical low-potent corticosteroid ointments. On the follow-up visit 12 months later nonpruritic hyperpigmentary changes of the same size and distribution were still present. The patient was advised to use emollients on a regular basis.

\section{Discussion}

Imatinib mesylate has often been implicated to various skin eruptions. Several types of adverse cutaneous reactions have been mentioned with imatinib, ranging from $9.5 \%$ to $69 \%$ in different cohorts [8]. Rash, edema, and pruritus appear most commonly and are usually described as moderate and dose-dependent [9]. At about $5 \%$ of cases are reported as severe and life-treatening. Extensive skin changes of grade 3 - 4 are Stevens-John-

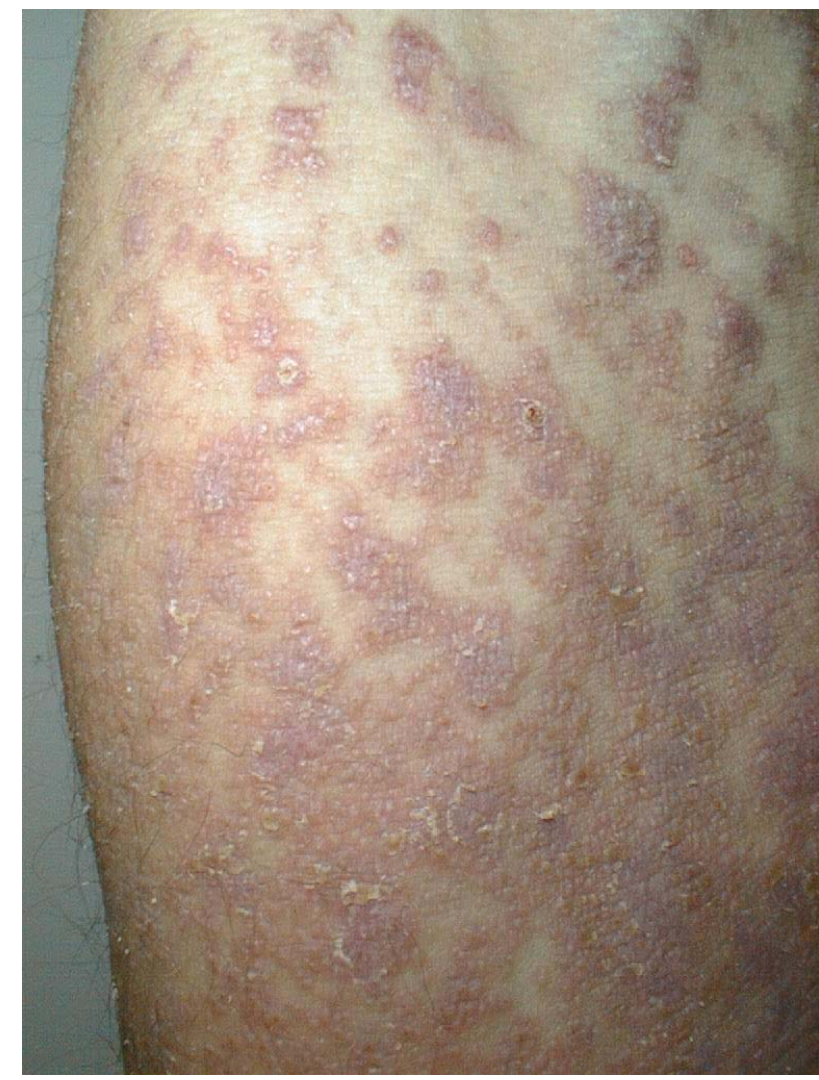

Figure 1. Extensive lichenoid reaction to imatinib.

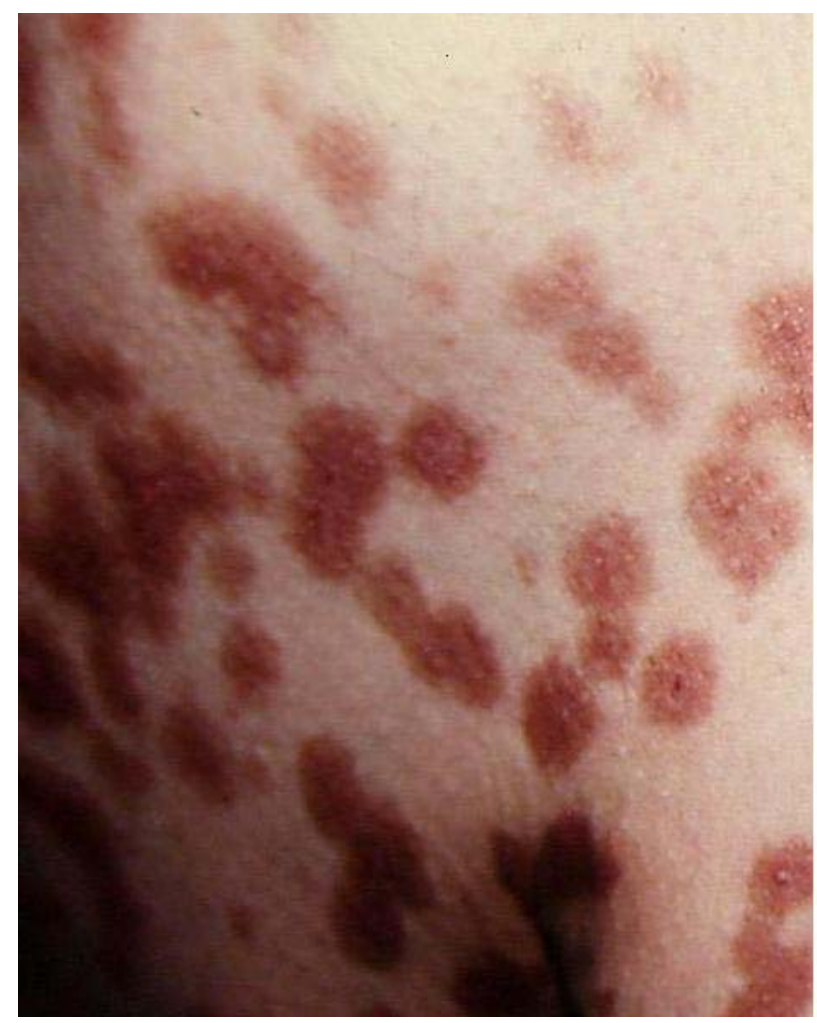

Figure 2. Wide spread plaques of hyperpigmentation on the patient's back. 


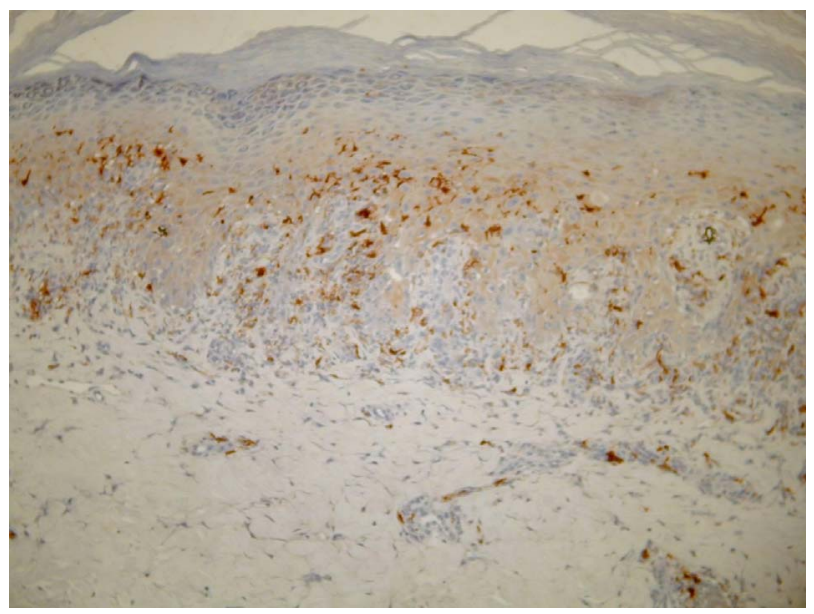

Figure 3. Parakeratosis, acanthosis, lichenoid lymphocytic infiltration with pigment incontinence in the upper dermis $[\mathrm{S100}, \times 100]$.

son syndrome, toxic epidermal necrolysis, acute generalized exanthematous pustulosis, vasculitis, and lichenoid eruption [10]. Such cases are most often accompanied by high fever, nausea, and gastro-intestinal involvement. Robert et al. reported a case of periorbital edema and hair depigmentation [11].

Cutaneous lichenoid eruption associated with imatinib is a rare adverse event [12]. Two of the four reported cases of lichenoid eruption due to imatinib involved the buccal mucosa and started 8 - 12 weeks after the first adminitration [13]. The molecular mechanisms of this side effect are still unknown. There are insufficient evidencebased data to establish guideline on its management. Due to the substantial clinical benefit, continuation of therapy is preferred while the skin rash is easily manageable with a short course of oral and topical corticosteroid. Temporary dose reduction or discontinuation of drug is required in the event of severe cutaneous eruptions. The current case had grade 4 toxicity including generalized skin eruption as well as high fever, malaise, and nausea. Permanent drug cessation was imposed by the progression of skin changes.

Nilotinib is considered in cases of imatinib resistance and intolerance. It was used as an alternative second-line therapy in our case. Nilotinib safety profile yields comparable results of rash occurrence with dasatinib [14]. It is rarely associated with dry skin and tolerable grade 1 2 skin rashes [15]. Lower incidence of skin rash (11\% and 15\%) was found in patients with blast crisis (myeloid and lymphoid, respectively), compared with $22 \%$ and $27 \%$ of the patients with accelerated and chronic phases of CML [16]. Two cases of Raynaud-like phenomenon induced by nilotinib have been recently reported [17]. No pigmentary skin changes induced by nilotimib have yet been described.

As small molecules of TKI gradually arise as CML treatments, the safety of these drugs becomes an important issue. Among imatinib-induced dermatologic side effects pigmentary changes are rarely reported. Interestingly, at about $40 \%$ of all reported cases are presented by hypopigmentation [18]. In one report, all seven patients had pre-existing skin eruption and developed post-inflammatory depigmentation [8]. However, Valeyrie L. et al. point out that only $5.4 \%$ of all patients with pigmentary changes had a pre-existing skin rash. Those authors suggest a complex molecular interaction in the pathogenesis of TKI pigmentary changes [1]. Speculation on germ-line mutations in kit, resulting in constitutive activation of the kit receptor with clinical manifestations of hyperpigmentation, has been made [19]. It is not known whether different germ-line mutations in kit lead to various clinical syndromes. Robson et al. observed hyperpigmentation in three out of seven families, which had the 559delV, V559A (two families), and W557R mutation of c-kit [19]. The presence of urticaria pigmentosa along with the other characteristics of this genetic syndrome is not surprising because systemic mastocytosis has been linked to disturbances in c-kit and imatinib is a successful treatment in such cases. Moreover, TKI favorable safety and efficacy profile in CML and gastro-intestinal stromal tumors emerges a promising role in the treatment of metastatic melanoma harboring the kit mutation [20]. The various interactions between photosensitivity and molecular pathways involved in the response to ultraviolet stress can explain the different phenotype of pigmentary changes caused by tyrosine kinase inhibitors. It seems that patient's original skin phenotype is also in close correlation. Pigmentation is associated with c-kit signaling and resultant activation of the tyrosine kinases involved in melanogenesis, such as tyrosinase and tyrosinase-related protein 1. Hemesath et al. suggested that the signal transmission downstream activation of the MAP kinase Erk-2, and modulate the pigment production through an effect on the tyrosine pigmentation gene promoter and expression of genes, essential for melanocyte survival and development [21].

\section{Conclusion}

In conclusion, we accentuate on the cross-reactivity of TKI and their possible role on modifying melanogenesis and melanocyte survival. This is a case of nilotinib-induced hyperpigmentation in a unique cross toxicity with imatinib, which had previously induced generalized lichenoid dermatitis in the same patient. In comparison with the previous standards of care, TKI benefits include more rapid response rates, increased survival, and fewer side effects. The improved long-term outcomes have altered the approach to CML management from a progressive fatal disease with a poor prognosis to a chronic condition similar to diabetes or hypertension. Prolonged sur- 
vival increases the need for patient education, support, monitoring, and assistance. Cutaneous side effects may adversely affect patients' quality of life and, therefore, require prompt attention to prevent long-term complications or suboptimal outcomes. Anecdotal case reports together with profound clinical trials can optimize regular monitoring and management of treatment-related side effects providing better therapy adherence and outcome.

\section{REFERENCES}

[1] L. Valeyrie, S. Bastuji-Garin, J. Revuz, N. Bachot, J. Wechsler, et al., "Adverse Cutaneous Reactions to Imatinib (STI-571) in Philadelphia Chromosome Positive Leukemias: A Prospective Study of 54 Patients," Journal of the American Academy of Dermatology, Vol. 48, 2003, pp. 201-206. doi:10.1067/mjd.2003.44

[2] S. G. O’Brien, F. Guilhot, R. A. Larson, et al., "Imatinib Compared with Interferon and Low-Dose Cytarabine for Newly Diagnosed Chronic Phase Chronic Myeloid Leukemia,” New England Journal of Medicine, Vol. 348, No. 11, 2003, pp. 994-1004. doi:10.1056/NEJMoa022457

[3] M. Schaich, K. Schakel, T. Illmer, G. Ehninger and M. Bornhauser, "Severe Epidermal Necrolysis after Treatment with Imatinib and Consecutive Allogeneic Hematopoietic Stem Cell Transplant," Annals of Hematology, Vol. 82, No. 5, 2003, pp. 303-304.

[4] M. Schwarz, K. A. Kreuzer, G. Baskaynak, B. Dorken and P. le Coutre, "Imatinib Induces Acute Generalized Exanthematous Pustulosis in Two Patients with Chronic Myeloid Leukemia,” European Journal of Haematology, Vol. 69, No. 4, 2002, pp. 254-256. doi:10.1034/j.1600-0609.2002.02830.x

[5] H. Kantarjian, F. Giles, N. Gattermann, K. Bhalla, et al., "Nilotinib (Formerly AMN 107), a Highly Selective bcrabl Tyrosine Kinase Inhibitor, Is Effective in Patient with Philadelphia Chromosome Positive Chronic Myelogenous Leukemia in Chronic Phase Following Resistance and Intolerance to Imatinib,” Blood, Vol. 110, 2007, pp. 35403546. doi:10.1182/blood-2007-03-080689

[6] M. Rios and P. Ault, "Identification of Side Effects Associated with Intolerance to BCR-ABL Inhibitors in Patients with Chronic Myeloid Leukemia,” Clinical Journal of Oncology Nursing, Vol. 15, 2011, pp. 660-667. doi:10.1188/11.CJON.660-667

[7] M. Lamchahab, M. Qachouh, F. Hali, H. Benchikhi, et al., "Successive Cutaneous Adverse Reactions to Nilotinib and Imatinibin a Single Patient," Annales de Dermatologie et de Venereologie, Vol. 139, No. 12, 2012, pp. 828831. doi:10.1016/j.annder.2012.09.015

[8] M. Brouard and J. H. Saurat, "Cutaneous Reactions to STI571,” New England Journal of Medicine, Vol. 345, No. 8, 2001, pp. 618-619. doi:10.1056/NEJM200108233450814

[9] B. J. Druker, F. Guilhot and S. G. O’Brien, “Five-Year Follow-Up of Patients Receiving Imatinib for Chronic Myeloid Leukemia,” New England Journal of Medicine, Vol. 355, No. 23, 2006, pp. 2408-2417.
doi:10.1056/NEJMoa062867

[10] L. T. Hsiao, H. M. Chung, J. T. Lin, T. J. Chiou, J. H Liu, et al., "Stevens-Johnson Syndrome after Treatment with STI571: A Case Report,” British Journal of Haematology, Vol. 117, No. 3, 2002, pp. 620-622. doi:10.1046/j.1365-2141.2002.03499.x

[11] C. Robert, J. C. Soria, A. Spatz, A. Le Cesne, et al., “Cutaneous Side-Effects of Kinase Inhibitors and Blocking Antibodies," Lancet Oncology, Vol. 6, No. 7, 2005, pp. 491-500. doi:10.1016/S1470-2045(05)70243-6

[12] C. Roux, A. M. Boisseau-Gersaud, I. Saint-Cyr, R. Hekenon, et al., "Lichenoid Cutaneous Reaction to Imatinib," Annales de Dermatologie et de Venereologie, Vol. 131, No. 6-7, 2004, pp. 571-573. doi:10.1016/S0151-9638(04)93669-1

[13] K. Prabhash and D. C. Doval, "Lichenoid Eruption Due to Imatinib," Indian Journal of Dermatology, Venereology and Leprology, Vol. 71, No. 4, 2005, pp. 287-288. doi:10.4103/0378-6323.16627

[14] C. Gambacorti-Passerini, T. Brummendorf, H. Kantarjian, et al., "Bosutinib (SKI-606) Exhibits Clinical Activity in Patients with Philadelphia Chromosome Positive CML or ALL Who Failed Imatinib,” Journal of Clinical Oncology, Vol. 25, 2007, p. 7006.

[15] Y. Wei, X. Zhang, W. Chen, R. Cao, C. Yin, R. Feng, Q. Liu and F. Meng, "Long-Term Outcomes of Nilotinib Treatment for Chronic Myelogenous Leukemia Patients with Imatinib Resistance or Intolerance,” Journal of Southern Medical University, Vol. 32, 2012, pp. 1000-1003.

[16] A. L. C. Agero, S. W. Dusza, C. Benvenuto-Andrade, et al., "Dermatologic Side Effects Associated with the Epidermal Growth Factor Receptor Inhibitors," Journal of the American Academy of Dermatology, Vol. 55, No. 4, 2006, pp. 657-670. doi:10.1016/j.jaad.2005.10.010

[17] C. Hazenberg, G. Ossenkoppele and W. Smit, "RaynaudLike Phenomenon in Two Patients on Nilotinib,” British Journal of Haematology, Vol. 158, No. 4, 2012, p. 431. doi:10.1111/j.1365-2141.2012.09215.x

[18] B. Arora, L. Kumar, A. Sharma, J. Wadhwa and V. Kochupillai, "Pigmentary Changes in Chronic Myeloid Leukemia Patients Treated with Imatinib Mesylate,” Annals of Oncology, Vol. 15, No. 2, 2004, pp. 358-359. doi:10.1093/annonc/mdh068

[19] M. E. Robson, E. Glogowski, G. Sommer, C. R. Antonescu, K. Nafa, R. G. Maki, et al., "Pleomorphic Characteristics of a Germ-Line KIT Mutation in a Large Kindred with Gastrointestinal Stromal Tumors, Hyperpigmentation, and Dysphagia," Clinical Cancer Research, Vol. 10, No. 4, 2004, pp. 1250-1254. doi:10.1158/1078-0432.CCR-03-0110

[20] A. Tran and H. Tawbi, "A Potential Role for Nilotinib in KIT-Mutated Melanoma,” Expert Opinion on Investigational Drugs, Vol. 21, No. 6, 2012, pp. 861-869. doi:10.1517/13543784.2012.679341

[21] T. J. Hemesath, E. R. Price, C. Takemoto, T. Badalian and D. E. Fisher, "MAP Kinase Links the Transcription Factor Microphthalmia to c-Kit Signalling in Melanocytes,” Nature, Vol. 391, No. 6664, 1998, pp. 298-301. doi:10.1038/34681 